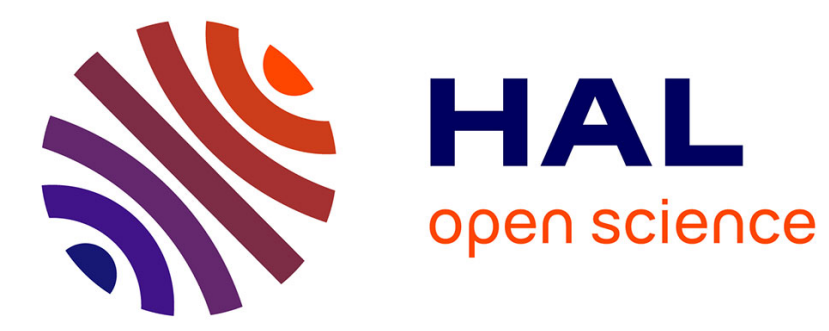

\title{
Thermodynamic origin of dendrite growth in metal anode batteries
}

\author{
Arthur Hagopian, Marie-Liesse Doublet, Jean-Sébastien Filhol
}

\section{To cite this version:}

Arthur Hagopian, Marie-Liesse Doublet, Jean-Sébastien Filhol. Thermodynamic origin of dendrite growth in metal anode batteries. Energy \& Environmental Science, 2020, 13, pp.5186. 10.1039/D0EE02665D . hal-03064653v2

\section{HAL Id: hal-03064653 \\ https://hal.science/hal-03064653v2}

Submitted on 17 Dec 2020

HAL is a multi-disciplinary open access archive for the deposit and dissemination of scientific research documents, whether they are published or not. The documents may come from teaching and research institutions in France or abroad, or from public or private research centers.
L'archive ouverte pluridisciplinaire HAL, est destinée au dépôt et à la diffusion de documents scientifiques de niveau recherche, publiés ou non, émanant des établissements d'enseignement et de recherche français ou étrangers, des laboratoires publics ou privés. 


\title{
Thermodynamic Origin of Dendrite Growth in Metal Anode Batteries
}

\author{
A. Hagopian, ${ }^{1,2}$ M.-L. Doublet, ${ }^{1,2} *$ J.-S. Filhol ${ }^{1,2} *$ \\ ${ }^{I} I C G M$, University of Montpellier, CNRS, ENSCM, Montpellier, France \\ ${ }^{2}$ RS2E French network on Electrochemical Energy Storage, FR5439, Amiens, France
}

*Corresponding authors: Marie-Liesse Doublet (marie-liesse.doublet@umontpellier.fr) and Jean-Sébastien Filhol

(jean-sebastien.filhol@umontpellier.fr)

\begin{abstract}
The formation of dendrites in alkali and alkaline earth-metal batteries leads to short circuit and catastrophic battery failure which hinders the development of high-energy-density battery technology. Herein, we investigate the thermodynamic origin of this complex phenomenon and show that kinetic transport limitation of metal cations in the electrolyte is not the only factor controlling the formation of dendrites. The specific behavior of $\mathrm{Li}, \mathrm{Na}$ and $\mathrm{Mg}$ electrodes towards dendritic growth is straightforwardly deduced from the shape of their electro-capillary diagrams, as computed from a grand canonical DFT approach. The whisker and dendrite morphologies associated with the different growth regimes are fully rationalized by the present methodology and the critical parameters controlling the dendritic growth on metallic surfaces are clearly identified. Further improving the description of the interface by means of a simplified yet realistic SEI built on carbonate-based decomposition products, we show that the over-potentials at which each growth regime is expected to occur can be predicted at a quantitative level, hence allowing the design of chemical strategies to prevent dendrite growth at metallic surfaces. More specifically, high surface tension associated with low surface capacitance and low potential of zero-charge is the target triptych to favor safe battery operation and can be obtained through appropriate chemical engineering.
\end{abstract}

\section{Introduction}

In order to satisfy high energy density applications such as electric vehicles and large-scale energy storage, lithium metal anode is being reconsidered owing to its high theoretical specific capacity (3860 $\left.\mathrm{mAh} . \mathrm{g}^{-1}\right)$ and low standard potential $(-3.04 \mathrm{~V} / \mathrm{SHE})$. The commercial use of lithium as anode material for high-energy-density batteries, including Li-S and hybrid Li-flow batteries, is unfortunately hindered by safety issues due to the formation of $\mathrm{Li}$ whiskers/dendrites upon cell charging. ${ }^{1,2}$ Whiskers growth can 
lead to irreversible capacity loss and short cycle life due to the presence of "dead lithium", but dendrites can also produce internal short circuits leading to thermal runaway and catastrophic battery failure. Despite the paramount role of Li dendrites in the battery failure, their formation is still poorly controlled and insufficiently understood to envision large-scale lithium metal batteries (LMBs) commercialization. Over the last decades, various experimental strategies were developed to safely utilize Li-metal anode in LMB technology. ${ }^{1-6}$ Attempts to suppress hazardous Li growth through the design of LiX alloys $(\mathrm{X}=\mathrm{C}$, $\mathrm{Si}, \mathrm{Sn}, \mathrm{Al}$, etc. $)^{7,8}$ surface functionalization ${ }^{9-13}$ or the development of solid-state electrolytes, ${ }^{4,14-18}$ have already enabled substantial improvements, but were unsuccessful to provide unique direction to control Li-dendrite growth which relies on multiple and interdependent factors. Indeed, Li growth in non-aqueous liquid electrolytes is controlled by various parameters such as current density, Li salt concentration, or electrolyte composition which are difficult to dissociate from one another in the different studies reported so far. ${ }^{4,10,19-23}$ The formation of Li-dendrites under various electrochemical conditions has revealed complex growth mechanisms. Bazant and coworkers reported distinct growth regimes over time evolution, associated with different over-potentials. ${ }^{22,24}$ As schematized in Fig.1, the first regime corresponds to the onset of Li-electrodeposition at a low over-potential during which Li cations plate the Li-metal surface in a rather uniform manner, leading to a stable pseudo-epitaxial growth regime. Monocrystalline lithium nanoparticles synthesized under $+0.1 \mathrm{~V} / \mathrm{Li}$ over-potential confirm the existence of this first regime. ${ }^{25}$ Upon this regime, salt concentration decreases close to the anode surface (due to kinetic limitations) which results in an over-potential to keep the current density constant. ${ }^{26}$ Depending on the applied current density, this over-potential ranges from +0.5 to $+3.5 \mathrm{~V} / \mathrm{Li}$ and triggers the second regime during which lithium grows as whiskers from the electrode surface. Such root-growing deposits (also called needle-like) keep a unidirectional feature without branching, and interweave with each other to form a global mosslike (or bush-like) pattern which is usually blocked by a nanoporous separator. ${ }^{22}$ Note that this mossy growth mechanism is observed for lithium ${ }^{22,27-29}$ and sodium ${ }^{29-32}$ but not for magnesium ${ }^{27}$ which suggests a dependence on the intrinsic nature of the metallic electrode. Finally, after a characteristic time called Sand's time, ${ }^{33,34}$ when the cation concentration reaches zero at the metal electrode, a drop of over-potential of ca. +1 volt is observed which does not depend on the current density and leads to Li-dendrite growth (also called tree-like) in a tip-growing manner. Note that the widely-used "dendrite" term -- which has been defined as a skeleton of a single crystal made of one stalk and branches ${ }^{35}$-- only applies to these branched patterns and results in a fractal-like growth that can penetrate the separator (in contrast to whiskers), leading to catastrophic failure, short-circuit or thermal runaway. ${ }^{22}$ This fractal growth was observed for lithium, ${ }^{22}$ sodium, ${ }^{31}$ and more recently for magnesium ${ }^{36,37}$ although the latter was initially thought to be immune to dendrite formation. 
Overall, these studies have enabled tremendous advances in the understanding and observation of Liwhiskers/dendrites, also clarifying consistent terminology for Li-growth in battery operating conditions. The striking differences in morphology and dynamics between the mossy and the dendritic growth were mostly interpreted as kinetically-driven processes -- namely a root-growth reaction-limited and a tipgrowth transport-limited process, respectively. ${ }^{22}$ However, a possible thermodynamic origin was not considered, though it might be a relevant asset to guide future developments of safer LMBs. Over the past five years, theoretical models were developed to address this question. They already provided valuable, yet partial information on surface properties directly or indirectly related to Li-growth such as Li diffusion in bulk electrolytes ${ }^{38-40}$ or on the electrode surface, ${ }^{41-47}$ Li charge-transfer ${ }^{48,49}$ and Li-dendrite patterns. ${ }^{47,50-}$ 52 In these approaches however, the impact of the electrochemical potential and solid electrolyte interphase (SEI) on the overall mechanism of Li-growth was not elucidated, although it is known to be critical.

To figure out the thermodynamic and microscopic origin of such a complex growth mechanism, a grand canonical approach in the density functional theory (DFT) framework is required to account for the potential-dependence of each growth regime. ${ }^{53}$ We propose an easy-handling computational method to address this question through the investigation of potential-dependent stability diagrams of metal-surfaces, hereafter referred to as electro-capillary diagrams. The surface environment of the metallic electrode is taken into account with an increasing degree of complexity from implicit solvent model (PCM) to more explicit description of the solid-electrolyte interphase (SEI). We show that the different growth regimes observed for each metal (metal $=\mathrm{Li}, \mathrm{Na}, \mathrm{Mg}$ ) can be rationalized in terms of surface intrinsic properties. The morphology of $\mathrm{Li}, \mathrm{Na}$ and $\mathrm{Mg}$ particles as-obtained from the present methodology is in full agreement with experimental observations and differs significantly from the one obtained without considering electrochemical conditions in the calculations. Criteria for the prediction of epitaxial, mossy and fractal growth regimes can then be extracted from the general shape of the computed electro-capillary diagrams and from crystal symmetry considerations. More specifically, we show that the formation of whiskers and dendrites can be directly linked to a phase transition in the metallic growth process associated with a symmetry breaking for some specific $(h k l)$-surfaces becoming more stable than the bulk (negative surface tension). Then, we rationalize the subtle differences observed for $\mathrm{Li}, \mathrm{Na}$ and $\mathrm{Mg}$ growth morphologies and show that a basic, yet reasonable description of the metal/SEI interface allows for a quantitative prediction of the over-potentials expected for each growth regimes. These results provide experimentalists with chemically meaningful parameters to prevent metallic growth phenomena. In particular, they support that anode materials with high surface tension, low potential of zero-charge and low surface capacitance are desirable to suppress metal whisker/dendrite formation. This approach paves the way for a systematic 
theoretical study of suitable electrolyte additives or innovative anode materials to design novel dendritefree batteries.

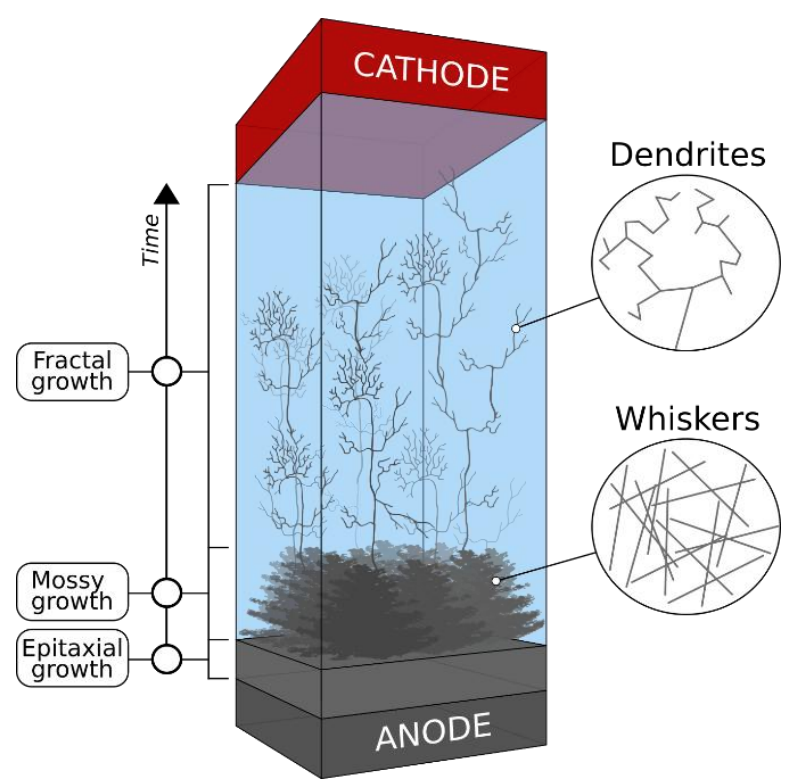

Figure 1: Schematic representation of lithium growth as observed experimentally under battery operating conditions. Three growth regimes are distinguishable over electroplating time evolution: first a stable pseudo-epitaxial growth followed by a mossy growth consisting in whiskers and finally a fractal growth consisting in dendrites. ${ }^{22}$

\section{Methodology}

To account for the potential-dependence of metallic-growth processes, we used a grand canonical DFT approach in which the free electrochemical energy is computed as a function of the electrode potential. ${ }^{53-55}$ The applied potential is varied through the addition of surface charges (positive or negative) which are counterbalanced by a homogeneous background charge to account for the electrical double layer response, following the method used in previous works (see Supplementary Information S1 for details). ${ }^{53,55-59}$ For solid electrodes, the surface tension $\gamma_{h k l}$ for a given $(h k l)$-surface is the work required to form a unit area of surface by cleaving the bulk, and its related free electrochemical energy, $F_{h k l}(E)$, can be directly extracted from DFT calculations, and linked to the surface tension $\gamma_{h k l}(E)$ through the relation:

$$
\gamma_{h k l}(E)=\frac{1}{2 A_{h k l}}\left(F_{h k l}(E)-N_{h k l} E_{b u l k}\right)
$$

In this expression, $2 A_{h k l}, N_{h k l}$ and $E_{b u l k}$ stand for the surface area of the two $(h k l)$-surfaces formed from bulk cleavage, the number of atoms in the slab layers, and the bulk reference energy (per atom), 
respectively. Introduced by Lippmann in $1875,{ }^{60}$ electro-capillary curves can be approximated at second order by the following expression:

$$
\Upsilon_{h k l}(E)=\frac{-C_{h k l}^{S}}{2}\left(E-E_{h k l}^{P Z C}\right)^{2}+Y_{h k l}^{P Z C}
$$

where $C_{h k l}^{S}, E_{h k l}^{P Z C}, Y_{h k l}^{P Z C}$ refer to the surface capacitance, the potential of zero-charge, and the surface tension at zero-charge of a $(h k l)$-surface, respectively, and where $E$ is the applied potential. As shown in Fig.2.a, a typical electro-capillary curve displays an inverted parabola shape, the top of which corresponds to the surface tension $\left(Y_{h k l}^{P Z C}\right)$ at zero-charge and the curvature of which is related to the surface capacitance (i.e. ability of the surface to delocalize charge-excess) ${ }^{61}$ which depends on the surface environment. The surface charge density $\sigma_{h k l}^{S}$ is then given by the Lippmann equation: ${ }^{62}$

$$
\sigma_{h k l}^{S}=-\left.\frac{\delta Y_{h k l}}{\delta E}\right|_{T, p,\left\{\mu_{i}\right\}}
$$

where $T, p$ and $\left\{\mu_{i}\right\}$ stand for the temperature, pressure and chemical potential, respectively. Equation (3) reflects the negative (resp. positive) surface charging for reductive (resp. oxidative) potentials lower (resp. higher) than $E_{h k l}^{P Z C}$. The classical inverted parabola shape of electro-capillary curves reflects the decrease of the surface tension induced by the surface charge-excess. In fact, the charge-induced coulombic repulsion decreases the cost of surface area increase (i.e. surface tension) as the increase of surface area dilutes the surface charge and thus stabilizes the surface. Hence, the larger the surface charge (positive or negative) the lower the surface tension (or equivalently the free electrochemical energy). Note that because cleavage is an endothermic process associated with bond breaking, the surface tension $\gamma_{h k l}$ is usually a positive quantity, and the surface area of a particle tends to be minimal relatively to the bulk volume as classically described by the Wulff approach. Nevertheless, surface-charge accumulation may lead to a critical electrode potential $\left(E_{h k l}^{c}\right)$ for which the electrostatic repulsion on the surface becomes so strong that the surface creation becomes energetically favored over bulk $(Y \leq 0)$. This scenario arises when the energy gain caused by the increase in surface area to reduce coulombic repulsions is larger than the energy cost of bond breaking required to cleave the bulk and form new surface area. This is highlighted in Fig.2.a, where $Y_{h k l}=0$ is achieved at a reductive potential $E_{h k l}^{c}$ below which the ( $h k l$ )surface area is expected to rapidly increase. The change in the $Y_{h k l}$ sign is therefore the signature of a phase transition in the metallic-growth process.

For a given metal $M$, the so-called M-electro-capillary diagram is obtained by the superimposition of different electro-capillary $(h k l)$-curves and dictates which $(h k l)$-surface first reaches the critical $\Upsilon_{h k l}\left(E_{M}^{c}\right)=0$ condition during the electrodeposition process. Note that the critical potential $E_{M}^{c}$ is an 
intrinsic property of the metal $\mathrm{M}$ and its environment, and corresponds to the highest $(h k l)$-critical potential $E_{h k l}^{c}$ associated to the first $(h k l)$-surface reaching the $Y_{h k l}=0$ condition. For the sake of clarity, we here introduce the critical over-potential, $\Delta E_{M}^{c}$, defined with respect to the metal standard potential as: $\Delta E_{M}^{c}=E_{M^{+} / M}^{0}-E_{M}^{c}$. The aim of the present work is to link the shape of the electro-capillary diagram, as computed in the grand canonical DFT approach, to the different regimes of metallic-growth, as experimentally observed, following the general methodology summarized below.
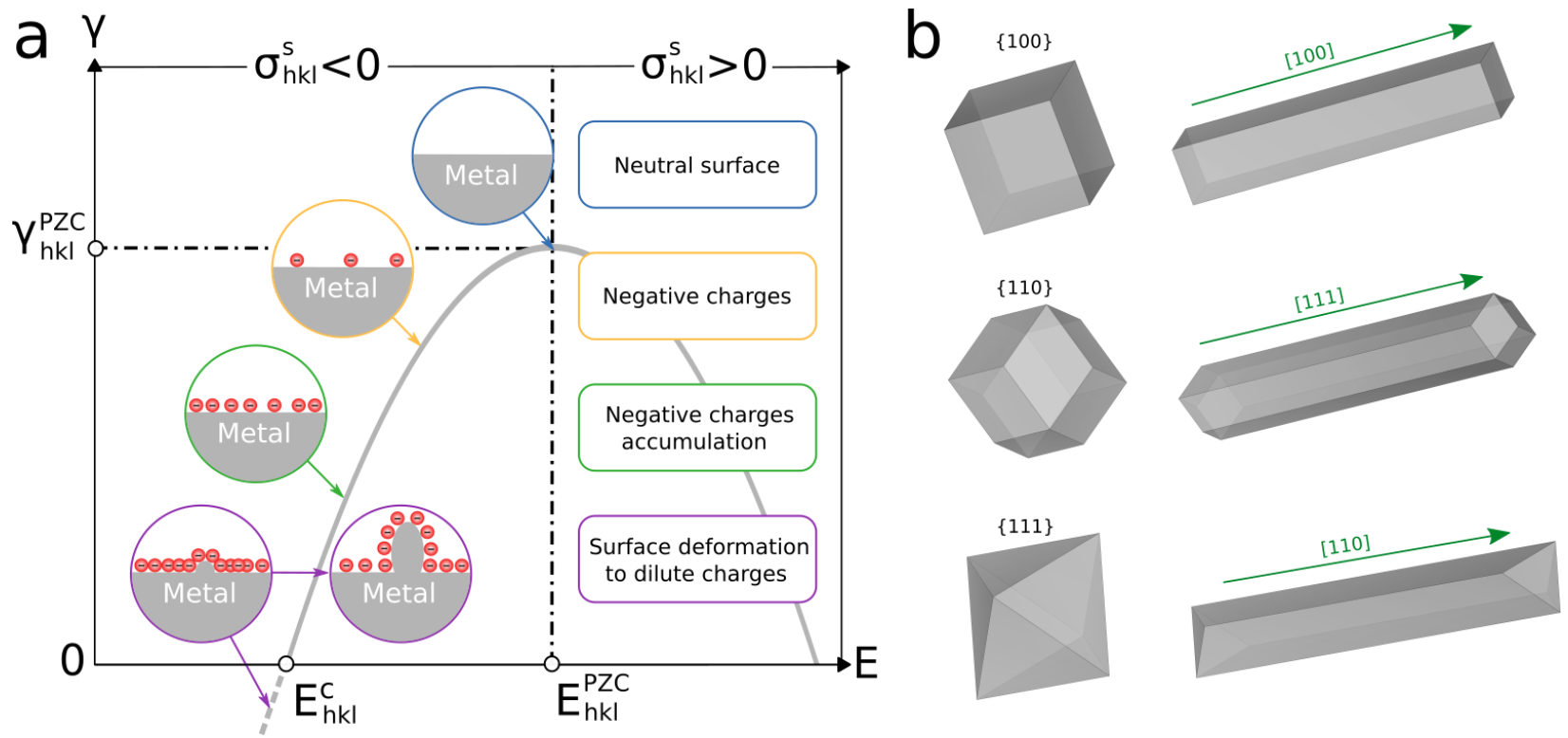

Figure 2: (a) Representation of an electro-capillary curve showing the general evolution of the (hkl)surface tension as a function of the applied potential E. The decrease in the (hkl)-surface tension with respect to the zero-charge state is due to the negative charge-excess induced by the decrease in potential with respect to the potential of zero-charge $E_{h k l}^{P Z C}$ (reductive condition). $E_{h k l}^{c}$ refers to the critical potential below which the (hkl)-surface becomes favored over bulk and should rapidly expand so as to minimize the particle free energy. (b) Illustration of anisotropic particle-growth induced by a symmetry lowering in one specific $\{h k l\}$-class (see Supplementary Information S2.A for details). Following the Miller terminology, (hkl), $\{h k l\}$ and $[h k l]$ define a plane, a set of symmetry-equivalent planes (class) and a surface direction in the basis of direct-lattice vectors, respectively.

Under reductive conditions $\left(E<E_{M^{+} / M}^{0}\right)$, electrodeposition is expected to take place when the metal surface Fermi level is high enough to reduce the solvated species of the electrolyte. ${ }^{53}$ For low overpotentials at which all $(h k l)$-surface tensions remain positive, electrodeposition leads to a pseudoepitaxial growth regime during which metallic-particles grow so as to minimize the total surface energy. The equilibrium shape of the particles, which consists in different $(h k l)$-surfaces at a given potential $\mathrm{E}$, 
can be obtained by building a Wulff construction from the potential-dependent surface tensions $Y_{h k l}(E)$ rather than the zero-charged ones $\left(Y_{h k l}^{P Z C}\right)$, as usually done. Therefore, at a given potential, the equilibrium crystal shape of the metallic-particles is deduced from the relative stability of all $(h k l)$-surfaces, the ones having the lowest surface tensions relatively to their orientation being dominant on the particle's facets. As surface tensions $Y_{h k l}(\mathrm{E})$ are potential-dependent, any change in potential altering the hierarchy in the $(h k l)$-surface tensions also modifies the particles morphology. For higher over-potentials, one $\{h k l\}$-class of symmetry-equivalent surfaces may reach the critical $Y_{h k l}\left(E_{M}^{c}\right)=0$ value for which surface-growth is favored over bulk. The critical over-potential $\Delta E_{M}^{c}$ associated to this specific critical potential value $E_{M}^{c}$ therefore points the end of the homogeneous three-directional epitaxial regime and the onset of a lowerdirectional regime during which metallic-particles preferentially grow so as to maximize their surface area along one specific $[h k l]$-direction. Noteworthy, for a high symmetry crystal structure, such lower directional particle-growth can only be achieved through a symmetry breaking of the bulk-crystal symmetry in order to prevent all surfaces belonging to the same $\{h k l\}$-class to expand simultaneously and identically. Possibly of kinetic origin, this symmetry lowering imposes that a surface subset of one given $(h k l)$-class expands more rapidly to the expense of the other $(h k l)$-surfaces, hence leading to strongly anisotropic particles (e.g. whisker-like, plate-like). Examples of unidirectional growth are given in Fig.2.b and a proof in a simple case of the energy stabilization induced by symmetry breaking is given in Supplementary Information S2.B. By extension, if more than one $\{h k l\}$-class of symmetry-equivalent surfaces simultaneously reaches the $Y_{h k l}(E) \leq 0$ condition, competitive unidirectional growth in various directions should result in a branched architecture growing in a multidirectional manner in order to maximize the global surface area over the bulk volume. Unless kinetic limitations slow down some growing directions over others, a fractal tree-like structure maximizing the surface area/volume ratio is expected when (almost) all ( $h k l)$-surfaces become energetically favored over bulk. In this context, the different regimes experimentally observed for metallic growth can be straightforwardly deduced from the general shape of each metal-related electro-capillary diagram.

\section{Results and Discussion}

The environment of lithium surfaces was first investigated with an implicit solvent parameterized with the dielectric constant of ethylene carbonate-based electrolytes, $\varepsilon=89.9$. All technical details for the interface models and DFT calculations are given in the Computational details section. The electro-capillary diagram built from the thirteen most relevant $\mathrm{Li}(h k l)$-surfaces ${ }^{63}$ is presented in Fig.3. All uncharged (hkl)surfaces have different surface tensions $Y_{h k l}^{P Z C}$ ranging from 29.08 to $33.47 \mathrm{meV} . \AA^{-2}$ (see Supplementary Information Table S1), in fair agreement with previous theoretical and experimental works. ${ }^{42,64,65}$ Their 
associated potential of zero-charge ranges from +1.24 to $+1.67 \mathrm{~V} / \mathrm{Li}$ and reflects the different surfacedipoles generated for each $(h k l)$ cleavage direction. As expected, the potential of zero-charge increases with the surface atomic density. ${ }^{66}$ For the $b c c$ lattice, the packing density $d_{h k l}$ increases as $d_{110}>d_{100}>$ $d_{111}$, consistently with $E_{110}^{P Z C}>E_{100}^{P Z C}>E_{111}^{P Z C}$. All curves present slightly different curvatures corresponding to surface capacitances $C_{h k l}^{S}$ at zero-charge ranging from 6.73 to $8.46 \mu \mathrm{F} . \mathrm{cm}^{-2}$, in reasonable agreement with available data for surfaces in organic carbonates media (ca. 6-15 $\mu F . \mathrm{cm}^{-2}$ ). ${ }^{67}$

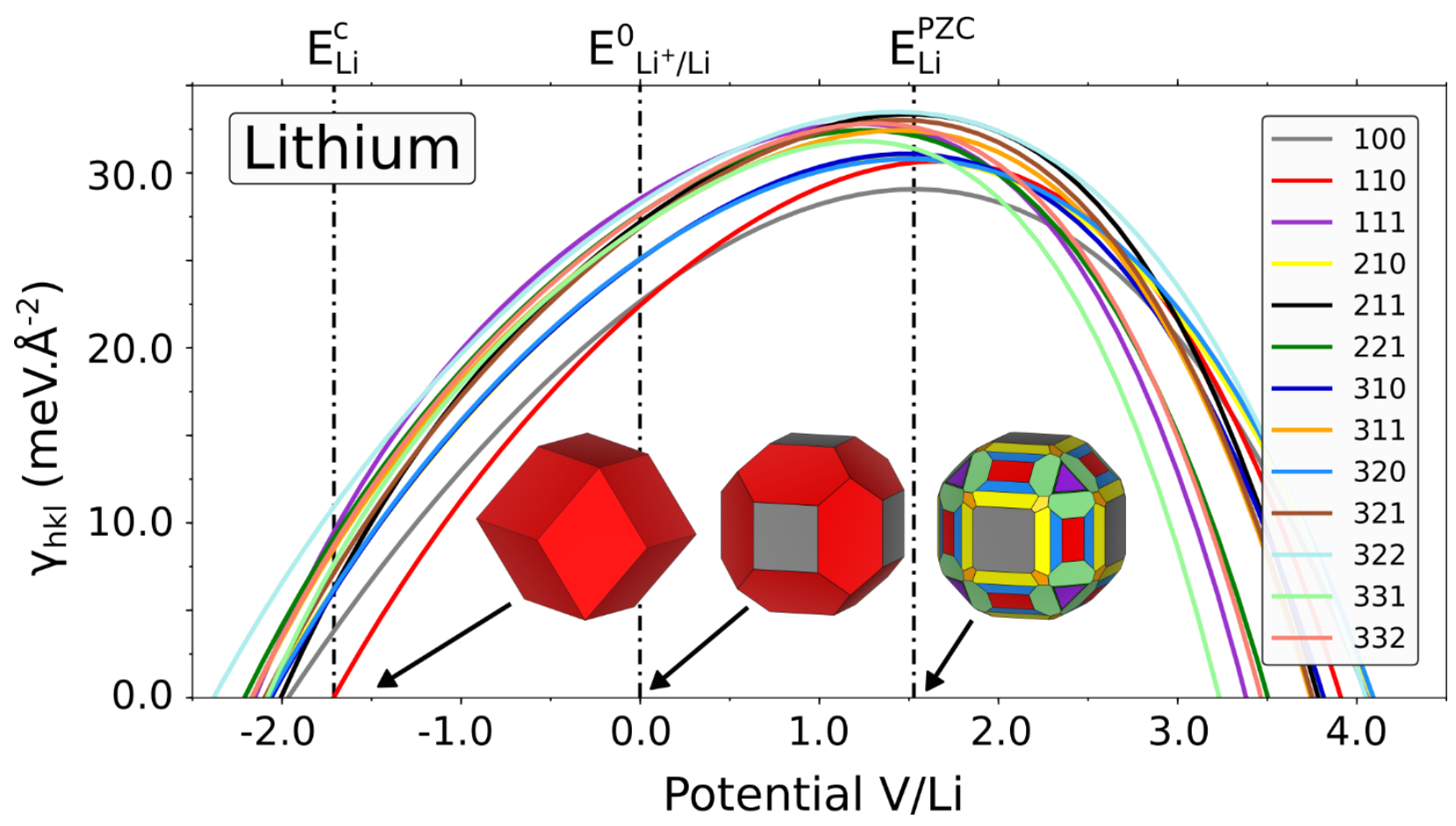

Figure 3: Lithium electro-capillary diagram as computed within the grand canonical DFT approach in PCM@Li conditions. $E_{L i}^{P Z C}=+1.53 \mathrm{~V} / \mathrm{Li}$ corresponds to the potential computed for the zero-charged Liparticles, using a Wulff construction for which the total charge of the particle (sum over all surfacecharges) is null. The critical potential $E_{L i}^{c}$ allows to determine the lithium critical over-potential defined as $\Delta E_{L i}^{c}=E_{L i^{+} / L i}^{0}-E_{L i}^{c}=+1.71 \mathrm{~V} / \mathrm{Li}$. The evolution of Li-particles shape, as obtained from the potentialdependent Wulff approach, is shown for various electrode potentials at $E=-1.50 \mathrm{~V} / \mathrm{Li}$ (reductive condition), $0 \mathrm{~V} / \mathrm{Li}$ and $+1.53 \mathrm{~V} / \mathrm{Li}$ (oxidative conditions).

The shape of Li-particles was predicted in various electrochemical conditions, following the electrocapillary diagram of Fig.3. To this aim, we used a Wulff construction based on the potential-dependent surface tensions $Y_{h k l}(E)$ computed at each potential of interest. ${ }^{68}$ An interesting asset of this procedure is that it allows one to deduce the potential of zero-charge of a Li-particle $\left(\mathrm{E}_{\mathrm{Li}}^{\mathrm{PZC}}\right)$ by constraining the total surface charge of the particle to be null (see Supplementary Information S3 for details). As previously 
shown, ${ }^{55}$ because of the Fermi level equilibration of all surfaces, a globally neutral particle may consists in positively and negatively charged surfaces. In the case of implicit solvation, $\mathrm{E}_{\mathrm{Li}}^{\mathrm{PZC}}$ is predicted at +1.53 $\mathrm{V} / \mathrm{Li}$, a potential at which Li-particles adopt a truncated cubic shape dominated by the (100) surface (Fig.3). This shape is in agreement with other DFT studies using uncharged surface energies. ${ }^{64,65}$ However, $\mathrm{E}_{\mathrm{Li}}^{\mathrm{PZC}}$ is far above the reference $\mathrm{Li}^{+} / \mathrm{Li}$ redox-pair potential (by definition $0 \mathrm{~V} / \mathrm{Li}$ ) so that $\mathrm{Li}$ particles are expected to be unstable towards the formation of oxidized $\mathrm{Li}^{+}$at this potential. The Wulff shape obtained using the uncharged standard approach corresponds to an unstable system under electrochemical conditions and is therefore not relevant. At a reductive potential of $E=-1.5 \mathrm{~V} / \mathrm{Li}$ a rhombic dodecahedron shape dominated by the $\{110\}$-surfaces is predicted by our calculations which significantly differs from the shape obtained at $\mathrm{E}_{\mathrm{Li}}^{\mathrm{PZC}}$. The consistency of this electrochemical approach can be checked by comparing the simulated particle shapes to experimental observations. Scanningelectron microscopy (SEM) data ${ }^{25}$ reveal that Li-nanoparticles display a rhombic dodecahedron shape with dominant $\{110\}$-surfaces at, however, moderate reductive potential $(-0.1 \mathrm{~V} / \mathrm{Li})$ compared to our predictions $(-1.5 \mathrm{~V} / \mathrm{Li})$. Hence, despite the relatively rough description of the Li-metal/electrolyte interface considered in our implicit model (PCM@Li), the present methodology leads to qualitative agreement with experiments. To which extent an explicit description of solvent molecules allows for a more quantitative description of the interface is a legitimate question. Previous studies have made such a comparison between implicit and explicit models. ${ }^{69,70}$ It was shown that including explicit water layers on metal surfaces influences the surface electric field but does not significantly impact the free energy associated with the different intermediates of $\mathrm{C}-\mathrm{C}$ bond formation mechanism during the electrochemical reduction of $\mathrm{CO}_{2}$. In this particular case, where explicit water molecules mainly add electrostatic interactions, the conclusion was that implicit approaches are sufficient to obtain quantitative results. Nonetheless, this observation is no longer valid in the situation where the inclusion of explicit molecules leads to strong chemical bonds between the solvent and the solute. As an example, we showed that an explicit description of lithium solvation shell in combination with a PCM is necessary to accurately evaluate lithium redox potential. ${ }^{57}$ This strongly suggests that a more realistic solvation model accounting for the chemical interactions between the Li-surface and the electrolyte is here required to predict redox potentials and make the present methodology quantitative.

To this aim, we explicitly included in our interface model a simplified, yet realistic description of the solid electrolyte interphase (SEI). SEI is known to form upon cell charging at the Li-surface and arises from partial electrolyte decomposition. It acts as a more or less passivating layer at the electrode/electrolyte interface which is typically nano- to micro-meter thick and protects the anode for further degradation due to high mechanical and electronic stability. For Li-anodes, SEI is described in the so-called "mosaic 
model" as a mixture of various insulating phases $\left(\mathrm{Li}_{2} \mathrm{O}, \mathrm{LiOH}, \mathrm{Li}_{2} \mathrm{CO}_{3}, \ldots\right)$ with carbonates as one of the dominant species at the metal/electrolyte interface. ${ }^{71,72}$ Therefore, different Li/carbonate interfaces (PCM@Li-CO $\mathrm{CO}_{3}$ ) were built (see Supplementary Information, Fig.S3 and Fig.S4) to check the impact of chemically modified Li-surfaces on the electro-capillary diagram of Fig.3. In order to limit computational cost, electrochemical calculations in explicit solvation were restricted to the four most stable Li $(h k l)$ surfaces. Details for PCM@Li-CO $\mathrm{CO}_{3}$ calculations are given in S4. As shown in Table S2, the potential of zero-charge of all $(h k l)$-surfaces is not significantly impacted by the carbonate adsorption. Accordingly, zero-charged Li-particles are found at very similar potentials in both implicit $\left(\mathrm{E}_{\mathrm{Li}}^{\mathrm{PZC}}=+1.53 \mathrm{~V} / \mathrm{Li}\right)$ and explicit conditions $\left(\mathrm{E}_{\mathrm{Li}}^{\mathrm{PZC}}=+1.56 \mathrm{~V} / \mathrm{Li}\right)$. Both values are consistent with the experimental work function measured for the Li-crystal $(+1.6 \mathrm{~V} / \mathrm{Li}){ }^{73}$ The impact of SEI is mainly seen in the surface tensions which are twice smaller for PCM@Li-CO 3 compared to PCM@Li due to the strong bonding between the carbonates and the metal surface. Interestingly, the (100) and (110) surface-tensions in explicit solvation are substantially different at $E_{L i^{+} / L i}^{0}$ while they were similar in implicit solvation. This can be easily linked to the surface packing density which discriminates the ability of the two surfaces to adsorb molecular species in terms of adsorption-sites and bonding strength. As a consequence, the fraction of each surface in the $\mathrm{Li}$ particle-shape is strongly modified in $\mathrm{PCM} @ \mathrm{Li}-\mathrm{CO}_{3}$ compared to PCM@ $\mathrm{Li}$ in such a way that the rhombic dodecahedron morphology experimentally observed at $-0.1 \mathrm{~V} / \mathrm{Li}$ is now accurately reproduced by our calculations at this potential (see Supplementary Information, Fig. S4). This quantitative agreement with experiments supports that the main electrochemical features of Li-SEI interfaces are reasonably well captured by our PCM@ $\mathrm{Li}-\mathrm{CO}_{3}$ model, provided that the capacitance is properly scaled (see Supplementary Information S4). It also stresses out that the evolution of Li-particles shape under electrochemical conditions has a thermodynamically origin, which persuaded us to further link our electro-capillary diagrams to the growth regimes experimentally observed for Liwhiskers/dendrites.

Under reductive conditions $\left(E<E_{L i}^{0} / L i\right.$ ) and for typical operating current-densities, $\mathrm{Li}^{+}$diffusion in bulk electrolyte may no longer compensate $\mathrm{Li}^{+}$consumption at the electrode/electrolyte interface which induces an over-potential to balance the electronic charge. As shown in Fig.4, three different over-potential domains leading to different electrode morphologies can be identified in the Li electro-capillary diagrams computed in implicit (PCM@Li) and explicit solvation (PCM@Li-CO ${ }_{3}$ ). As far as over-potentials are low enough so as all $\Upsilon_{h k l}$ are positive $\left(E_{L i}^{c}<E<E_{L i^{+} / L i}^{0}\right)$, Li-particles are expected to grow in a pseudoepitaxial regime to minimize their total surface area and free energy. In this domain (green region in Fig.4), our calculations predict the (110)-surface as the most stable Li-surface so that Li-particles are expected to grow with a rhombic dodecahedron morphology dominated by the (110)-surface, in perfect 
agreement with experimental observations. ${ }^{25}$ As discussed above, explicit solvation is required to access quantitative prediction of the absolute $\Delta E_{L i}^{c}$ over-potential at which the epitaxial regime ends. Below the $\Delta E_{L i}^{c}=+0.65 \mathrm{~V} / \mathrm{Li}$ predicted for the PCM@ $@ \mathrm{Li}-\mathrm{CO}_{3}$ interface, a second domain is visible in our electrocapillary diagrams (blue region in Fig.4) upon which solely the (110)-surface displays a negative surface tension $Y_{110} \leq 0$. This potential window corresponds to a second growth-regime characterized by a symmetry breaking of the equilibrium crystal shape to minimize the particle free energy and favoring lowdirectional growth. Kinetics or size effects may be an important factor in the crystal shape, but their impact is difficult to determine at this stage. Nevertheless, only three over all possible symmetry lowering in the $\{110\}$-class lead to $\{110\}$-faceted mono-crystalline objects growing in the [111]-direction (see Supplementary Information, Fig.S5), as experimentally observed. ${ }^{25,72}$ The hexagonal and triangular cross sections obtained with this model are also in agreement with experimental observations. Noteworthy, any other $(h k l)$-surface reaching the critical $Y_{h k l}\left(E_{L i}^{c}\right)=0$ would induce different shapes and growthdirections, therefore supporting the consistency of the present approach to rationalize the characteristic whisker shape and its unidirectional spatial expansion which both form the mossy aggregate experimentally observed. Interestingly, not only the whisker's shape is precisely determined by our thermodynamic approach, but also the over-potential at which this peculiar growth-regime starts agrees with experiments. Indeed, according to Bazant's observations, ${ }^{22}$ mossy growth is observed for an overpotential of $\sim 0.4-0.5$ volt at low current density $\left(\sim 1 \mathrm{mAh} / \mathrm{cm}^{2}\right)$. Although more sophisticated interfacemodels with more exhaustive descriptions of the SEI could even improve our results, the present prediction of $\Delta E_{L i}^{c}=+0.65 \mathrm{~V} / \mathrm{Li}$ is only slightly over-estimated compared to the experimental value, hence attesting that both the methodology and the interface model are relevant. Eventually, the third and last regime corresponding to Li-dendrite growth is shown to occur experimentally after a +1 volt overpotential drop which is now independent of the applied current density. Such a potential drop corresponds in our electro-capillary diagrams to a third domain (indicated in red in Fig.4) in which most or all (hkl)surfaces have reached the critical $Y_{h k l}(E)=0$ value. This regime is therefore very consistent with the growth of branched architectures (fractal growth characteristic of dendrite shape) resulting from the removal of the unidirectional symmetry constraint associated with the whiskers growth.

Overall, the present methodology allows good estimates of the relative stability of Li-surfaces under electrochemical conditions, which in turns controls the evolution of the particle shape and triggers specific Li-dendrite growth-regimes. In contrast with current knowledge, our results suggest that Li-dendrite growth is not only kinetically-driven by $\mathrm{Li}^{+}$diffusion limitation but also has a thermodynamic origin through the $Y_{h k l}(E)$ surfaces tensions that govern the Li morphology under electrochemical conditions. To 
support this hypothesis, we extended the present study to other metallic electrodes such as $\mathrm{Na}$ and $\mathrm{Mg}$ for which experimental observations are also available.

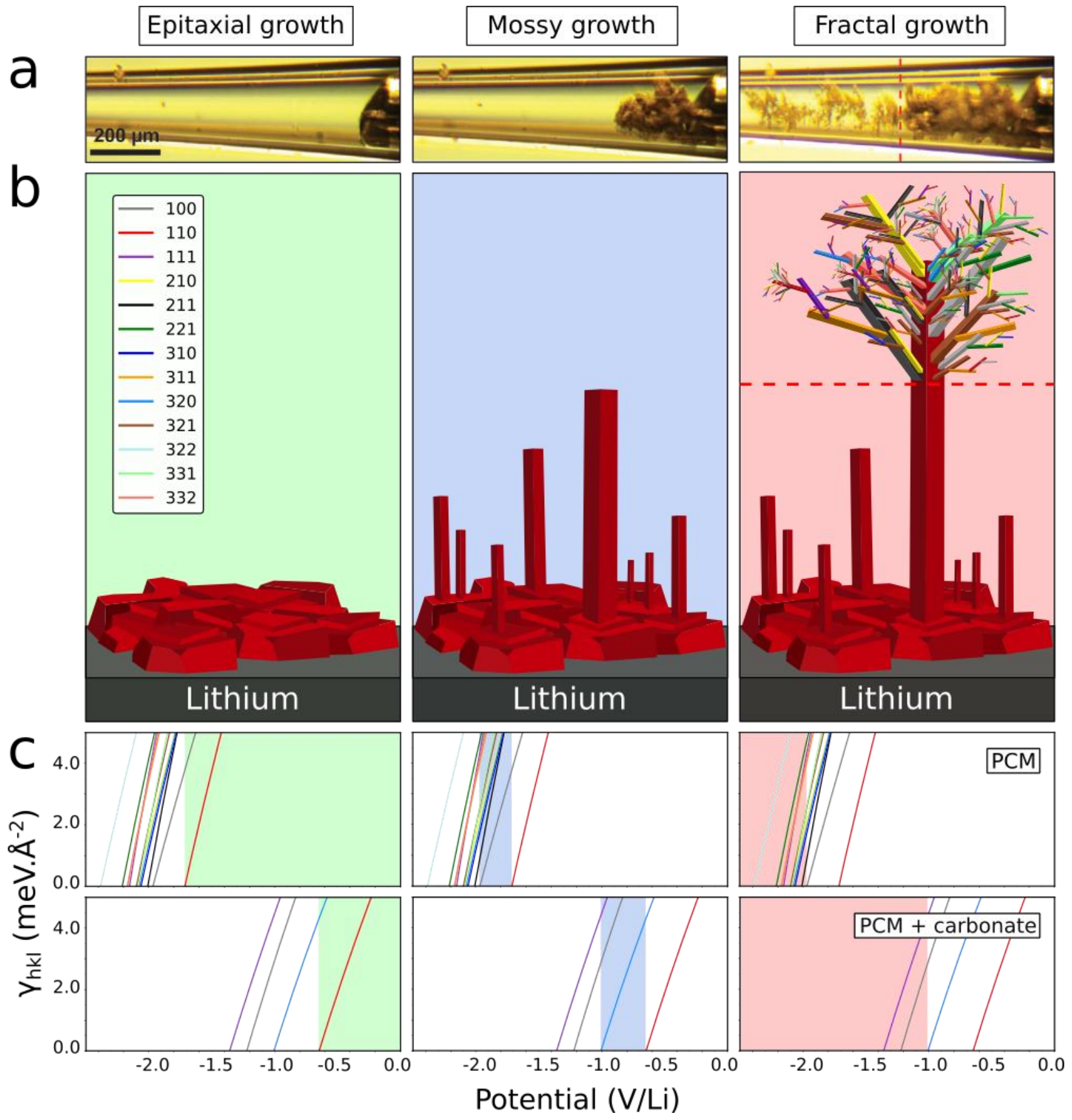

Figure 4: (a) Lithium growth regimes as experimentally observed ${ }^{22}$ - Published by the Royal Society of Chemistry. (b) Schematic representation of the different lithium growth regimes expected from (c) the electro-capillary diagrams computed in implicit (PCM) and explicit (PCM + carbonate) conditions for the Li electrode, and magnified in the potential region of interest (reductive conditions, $E<E_{\mathrm{Li}^{+} / \mathrm{Li}}^{0}$ ). The 
green, blue and red domains define the potential-window predicted for the epitaxial, mossy and fractal growth, the width of which is directly linked to the over-potentials at which each growth-regime starts (experimental observations). Results show that implicit solvation is sufficient to qualitatively predict and fully rationalize the different growth mechanisms while explicit solvation also enables quantitative predictions of the over-potentials experimentally measured for the different Li-growth regimes.

The same methodology was applied to sodium and magnesium, both being thought as promising alternative to lithium for the next generation of rechargeable metal batteries, while also undergoing undesirable and uncontrolled dendrite growth under specific operating conditions. ${ }^{29-32,36,37}$ As mentioned in the introduction, sodium presents a two-step growth instability process equivalent to the mossy and fractal growth reported for $\mathrm{Li}$, while magnesium presents a first growth-regime as thin hexagonal platelets formation, then followed by hazardous dendrites. Sodium and magnesium electrodes were considered in PCM conditions with a dielectric constant corresponding to classical solvents, ethylene-carbonate-based ( $\varepsilon$ $=89.9)$ and ethereal-based $(\varepsilon=7.2)$, respectively. Their electro-capillary diagrams and potentialdependent particle shapes are shown in Fig.5. Also having a bcc lattice, Na behaves similarly as lithium under reductive conditions and presents the same rhombic dodecahedron Wulff shape at $-1 \mathrm{~V} / \mathrm{Na}$. Na electro-capillary diagram computed in PCM (Fig.5.a) shows the onset of a $\{110\}$-dominated unidirectional growth regime, similar to $\mathrm{Li}$. The formation of Na-whiskers leads to mossy-like growth which, however, starts at a slightly lower over-potential than for $\mathrm{Li}\left(\Delta E_{N a}^{c}=+1.37 \mathrm{~V} / \mathrm{Na}\right.$ compared to $\Delta E_{L i}^{c}$ $=+1.71 \mathrm{~V} / \mathrm{Li}$, as computed in PCM conditions). For Mg (Fig.5.b), the most stable surface of the hcp lattice is the (0001) leading to a hexagonal crystal shape, irrespectively of the potential. For reductive potentials at which all $\gamma_{h k l}$ remain positive, the increase of the (0001) fraction induces a flattening of the particle shape as experimentally observed. ${ }^{59}$ At $\Delta E_{M g}^{c}=+2.42 \mathrm{~V} / \mathrm{Mg}$ over-potential, the $\{0001\}$-class consisting in only two $(0001)$ and $(000 \overline{1})$ surfaces reaches the $Y_{h k l}(E)=0$ critical surface tension. The increase of one surface area necessarily leads to the increase of the other one, which results in the formation of thinner and thinner hexagonal platelets (Fig.5.d). Accordingly, our calculations confirm that $\mathrm{Mg}$ does not undergo unidirectional whisker formation, alike $\mathrm{Li}$ and $\mathrm{Na}$, but a bidirectional growth of thin hexagonal platelets. This growth is slower than the unidirectional growth of whiskers, as the particle expansion is proportional to the inverse of the hexagon radius, and does not lead to a mossy layer but to interspersed thin hexagonal platelets, fully consistent with experimental observations. ${ }^{37}$ 

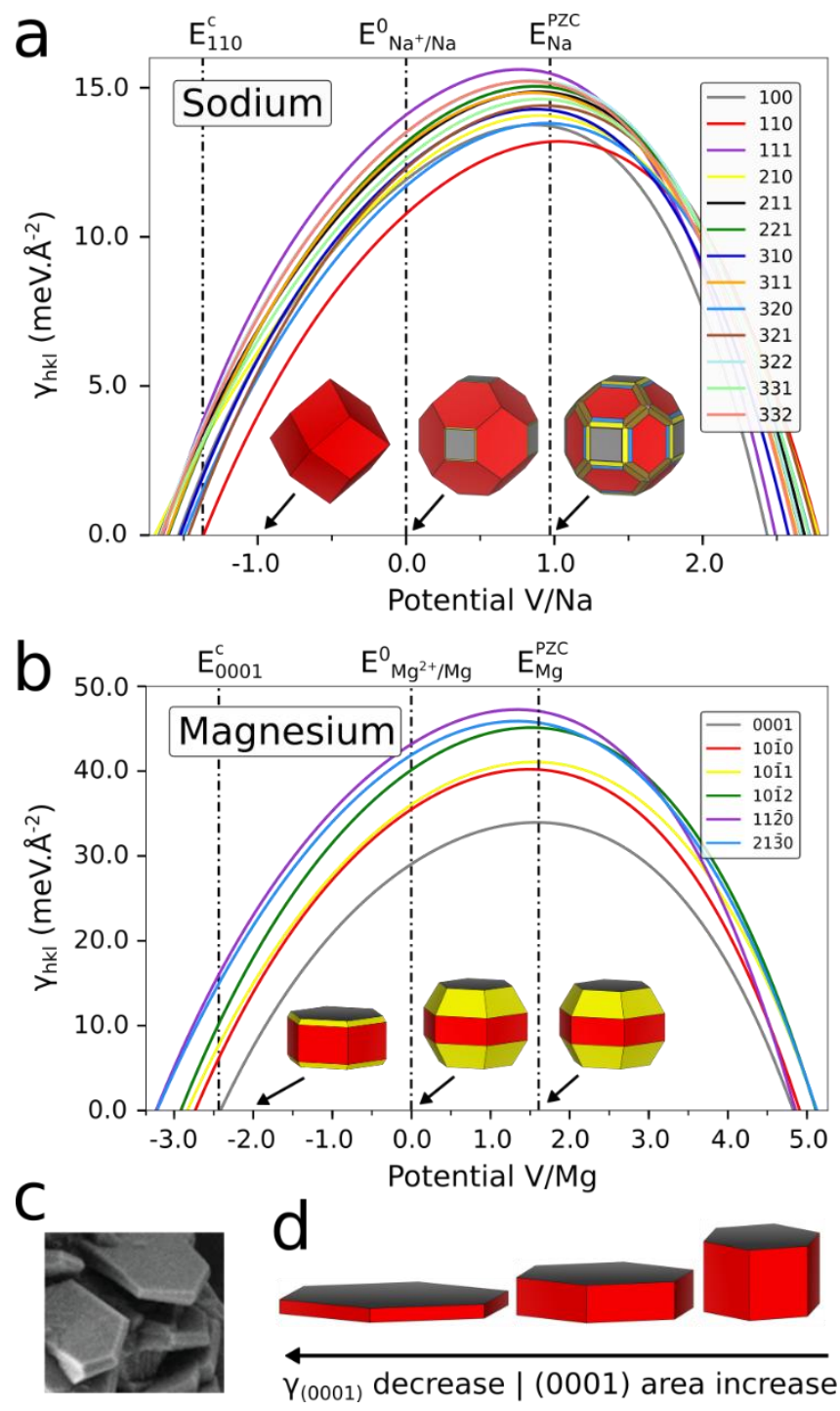

Figure 5: (a) Sodium electro-capillary diagram. (b) Magnesium electro-capillary diagram. (c) Experimental observation of magnesium thin hexagonal platelets, adapted from Ref.[37] with permission from The Royal Society of Chemistry. (d) Magnesium Wulff constructions simulated with (0001) surface tension decreasing.

$\mathrm{Li}, \mathrm{Na}$ and $\mathrm{Mg}$ critical over-potentials computed in PCM conditions follow $\Delta E_{M g}^{c}>\Delta E_{L i}^{c}>\Delta E_{N a}^{c}$, suggesting that 1) the pseudo-epitaxial domain is more extended for $\mathrm{Mg}$ than for $\mathrm{Li}$ and $\mathrm{Na}$ and the surface instability is more difficult to reach for $\mathrm{Mg}$, and 2) $\mathrm{Na}$ is slightly more prone to whisker/dendrite growth than $\mathrm{Li}$ and $\mathrm{Mg}$. As mentioned above, these critical over-potentials computed in PCM conditions are over estimated due to the lack of explicit chemical bonding at the metal surface which artificially increases the surface tensions (Supplementary Information table S1 and table S2). However, the adsorption energies of $\mathrm{CO}_{3}{ }^{2-}$ species, $\mathrm{E}_{\mathrm{CO}_{3}^{2-}}^{\text {ads }}$, computed on $\mathrm{Li}, \mathrm{Na}$ and $\mathrm{Mg}$ dominant surfaces are nearly 
constant (Supplementary Information table S5) and show that surface tensions are decreased by approximately the same factor for $\mathrm{Li}$ (51\% for $\mathrm{Li}$ (110)-surface), $\mathrm{Na}$ (44\% for $\mathrm{Na}$ (110)-surface) and $\mathrm{Mg}$ (55\% for $\mathrm{Mg}$ (0001)-surface) when identical carbonate density is considered. This supports that explicit solvation mainly impacts surface tension (within the same order of magnitude) and allows rapid evaluation of $\mathrm{Na}$ and $\mathrm{Mg}$ critical over-potentials, which are here found to be $\Delta E_{N a}^{c}=+0.59 \mathrm{~V} / \mathrm{Na}$ and $\Delta E_{M g}^{c}=+1.42 \mathrm{~V} / \mathrm{Mg}$, following the same order $\Delta E_{M g}^{c}>\Delta E_{L i}^{c}>\Delta E_{N a}^{c}$ than in PCM conditions. This makes the purely implicit model a reasonable model to compare critical over-potentials of alkali, alkaline-earth and their alloys.

For high enough current-density, increased cation consumption causes depletion in the electrolyte close to the metallic surface which ultimately leads to dendrite formation, as described above. Lithium, sodium and magnesium having close capacitances (Supplementary Information, Table S1, S3 and S4), the difference in their $\Delta E_{M}^{c}\left(\Delta E_{M g}^{c}>\Delta E_{L i}^{c}>\Delta E_{N a}^{c}\right)$ mainly arises from the difference in their surface tensions $\left(Y_{0001}^{P Z C}(M g)>Y_{110}^{P Z C}(L i)>Y_{110}^{P Z C}(N a)\right)$ and potentials of zero-charge $\left(E_{N a}^{P Z C}<E_{L i}^{P Z C}<E_{M g}^{P Z C}\right)$ which are therefore the two thermodynamic quantities one should first tune to avoid dendrite formation. From the expression of surface tension given in eq. (2) the critical over-potential $\Delta E_{M}^{c}$ above which a metal $\mathrm{M}$ is prone to whiskers/platelets formation can be estimated with respect to its standard potential $E_{M^{+} / M}^{0}$ from the zero-charge properties following:

$$
\Delta E_{M}^{c} \approx \sqrt{\frac{2 r_{h k l}^{P Z C}}{C_{h k l}^{S}}}-E_{h k l}^{P Z C}+E_{M^{+} / M}^{0}
$$

Thus, increasing the critical over-potential to prevent whisker or dendrite growth could be achieved by appropriate chemical engineering of the metal surface in order to maximize the surface tension, minimize the surface capacitance or shifting the potential of zero-charge towards lower values. Hence, to prevent dendrite growth, an optimal SEI material should satisfy the following conditions: 1) minimize solvent decomposition and carbonate formation, 2) be stable over decomposition even with direct contact with the Li-surface, 3) make weak bonds with the Li-surface to reduce surface tension, 4) have a low surface dipole to decrease the potential of zero-charge, 5) be an electronic insulator to reduce the surface capacitance and 6) be a lithium conductor to allow $\mathrm{Li}^{+}$transport and reduction. Turning back to the experimental and theoretical approaches so far developed to suppress dendrite formation, we can now fully rationalize their impact on dendrite formation with respect to these different parameters. First, in the Li case, the carbonate-based SEI is stabilizing the metal surface, ${ }^{74}$ decreasing its surface tension and potential of zerocharge, therefore favoring dendrite formation. Hence, although SEI is known to be essential for Li-ion battery operation, it also appears as one of the major causes of dendrite growth instabilities. Preventing 
alkyl carbonates decomposition on the metal surface could constitute a winning strategy to postpone or suppress such instabilities. In previous works, ${ }^{57,75}$ we have shown that alkyl carbonate solvents coordinated to $\mathrm{Li}^{+}$or $\mathrm{Mg}^{2+}$ cations undergo an electrophilic activation under reductive conditions leading to solvent decomposition and carbonates release. It is therefore not surprising that superconcentrated electrolytes decrease the ability of solvent to decompose at the metal surface ${ }^{10}$ as they favor ion-pairing. At high salt concentrations, the probability that cations have one counterion in their first solvation shell is very high, thus quenching the electrophilic activation of alkyl carbonates and hopefully dendrite growth. A less extreme solution to obtain dendrite-free surfaces is to use conventional electrolytes with specific additives $^{1}$ favoring the formation of SEI less bonded to the Li surface than carbonates. One example is the fluorination of the Li electrode which generates thick enough interfaces with a higher surface tension, thus allowing few hundreds of battery cycles ${ }^{12}$ without dendrite formation. Another example is the use of 2D atomic crystals, such as $h$-BN, deposited on the electrode surface. ${ }^{76,77}$ This interfacial layer allows a uniform deposition of metallic cations due to its low diffusion barrier and high stiffness. On top of this kinetic impact, it has been shown that $h$-BN layer interacts with the anode surface through weak van der Waals interactions which leads to high surface tension and therefore important critical over-potential. This thermodynamic impact is in perfect coherence with our model. Finally, the use of cesium as a plating additive to reduce dendrite formation was described in the self-healing electrical shielding mechanism. ${ }^{13}$ Cs atoms can be incorporated at low over-potential in the Li metal interface $\left(\mathrm{Cs}^{+}\right.$is reduced $0.1 \mathrm{~V}$ before $\mathrm{Li}^{+}$) to form an alloy-surface. As a highly polarizable atom, Cs should be more stable at the interface than in the Li-bulk, therefore staying at the interface. This should drastically decrease the binding energy of the Cs-surface with carbonates therefore increasing the surface tension. Furthermore, as Cs is slightly more electropositive than $\mathrm{Li}$, the surface dipole should be reduced, so as the potential of zero-charge.

All these examples show that optimizing each key-parameter independently of the other is complex as they all tend to be interdependent. Thus, a solution to the dendrite growth issue could be to use bifunctional molecules associated with separated adsorption and polarization moieties for pre-coating the electrode surface. The first functionalized group would control the Li-surface binding and thus the surface tension while the second polar group would control the surface dipole and thus the potential of zerocharge. By this means, one could play independently on the surface tension and potential of zero-charge, thus offering subtle mastering of the interface for more efficient prevention of dendrite growth. Nowadays, the main challenge we face is that strategies for dendrite prevention must be effective on longterm cycling, whereas Li-particle volume change upon charge and discharge inevitably leads to strong mechanical strain on the coating layer. On the one side, non-sticking hard layers should favor high surface tension but it is likely that the coating will undergo undesired cracks, which ultimately open channels for parasitic reactions of the uncovered Li-electrode with electrolyte. On the other side, soft materials such as 
polymers should easily decompose in contact with the highly reductive Li-surface, thus losing their mechanical protection. So, one possible strategy could be to mix one first layer of non-sticking hardcarbon ( $h$-BN-like) in direct contact with $\mathrm{Li}$, to increase the critical over-potential of Li-dendrite formation, with one second layer of soft functionalized polymers, to accommodate the mechanical stress while still insuring $\mathrm{Li}^{+}$transport across the layers. Furthermore, excess amount of Li-metal associated with large particle size could be used to reduce the volume change and the stress on the coating layer. Whatever is the strategy developed in the near future, the methodology presented therein can efficiently screen in silico target molecules and/or layers before being experimentally tested in real conditions.

\section{Conclusion}

Growth mechanisms of $\mathrm{Li}, \mathrm{Na}$ and $\mathrm{Mg}$ were investigated by means of a thermodynamic grand canonical DFT approach accounting for the potential-dependence of surface tensions. Results highlight the thermodynamic origin of the epitaxial, mossy and fractal growth regimes that are linked with intrinsic energetic properties of metal surfaces. The metal surface instability under reductive conditions is characterized by an over-potential threshold $\Delta E_{M}^{c}$ above which surface charge repulsions overcome the work required to increase the surface area. This critical $\Delta E_{M}^{c}$ sets the reductive potential below which one (at least) metallic surface becomes more stable than the bulk (negative surface tension). By having the lowest critical over-potential, $\mathrm{Na}$ is more prone to whisker/dendrite growth than $\mathrm{Li}$ and $\mathrm{Mg}$. At this critical over-potential, the equilibrium crystal shape switches from a surface area/volume minimization to a surface area/volume maximization to dilute the surface charges. For both $\mathrm{Li}$ and $\mathrm{Na}$, this implies a symmetry breaking in one $\{\mathrm{hkl}\}$-class of surfaces leading to unidirectional growth of whiskers. For the $h c p-\mathrm{Mg}$, a bidirectional growth resulting in thin hexagonal platelets is obtained to maximize the (0001)surface area. As the over-potential increases, a negative surface tension is achieved for more (hkl)-surface orientations, thus leading in the end to fractal growth. Thanks to this study, three interdependent keyparameters inherent to the metal surface properties are identified as tunable quantities to prevent dendrites: the surface capacitance, the surface tension that needs to be increased through the formation of SEI less strongly bonded to the metal surface than carbonates, and the potential of zero-charge that needs to be lowered through the decrease of the surface dipole. By providing qualitative to quantitative estimates of these three thermodynamic parameters, we believe the present methodology is a valuable tool to systematically screen novel families of multi-functionalized additives to master dendrite-free electrochemical interfaces for safe and high-energy-density metal batteries.

\section{Computational details}


Periodic calculations were performed within the density functional theory (DFT) framework, using the Vienna ab initio simulation package (VASP) ${ }^{78-81}$ and the projector augmented wave (PAW) method, ${ }^{82,83}$ as implemented in VASP. The exchange-correlation effects have been accounted for by the generalized gradient approximation (GGA) using the functional of Perdew, Burk and Ernzerhof (PBE) ${ }^{84}$ The electronic wave functions have been defined considering a plane-wave basis set up with an energy cutoff of $450 \mathrm{eV}$ for every metals. The Brillouin zone integration for $\mathrm{Li}, \mathrm{Mg}$, and $\mathrm{Na}$ bulks have been performed with 14x14x14 Gamma centered k-point mesh. This reference k-point set has been adapted for slab systems, depending on lattice parameters of every cleavage directions. For geometry optimization, convergence criterions have been set to $10^{-6} \mathrm{eV}$ and $10^{-2} \mathrm{eV} . \AA^{-2}$ respectively for electronic iterations and ionic relaxation. For all calculations in PCM, the vacuum thickness in the z-direction has been set to $15 \AA$, 7 atomic layers have been defined and the lattice parameters have been fixed as well as the central layer. For PCM@Li- $\mathrm{CO}_{3}$ calculations, the vacuum thickness was upscaled to $25 \AA$. An implicit solvent has been used as implemented in the patch VASPSol ${ }^{85}$ to simulate the presence of the most commonly used solvents for each metal in their respective battery conditions: permittivity of the solvent has been set to 89.9 (ethylene carbonate (EC)) for $\mathrm{Li}$ and $\mathrm{Na}$, and 7.2 (dimethoxyethane (DME)) for $\mathrm{Mg}$. The cavitation energy contribution has been set to $0 \mathrm{meV} . \AA^{-2}$, and the cutoff charge density parameter (which determines at which value of the electron density the dielectric cavity forms) has been set to $2.5 \cdot 10^{-4} \AA^{-3}$ and $5.10^{-5} \AA^{-3}$ for Li in PCM@Li and PCM@Li-CO 3 respectively, 1.25.10-4 $\AA^{-3}$ for Na and 5.10 $0^{-4} \AA^{-3}$ for Mg. Details on the PCM parameters can be found in Ref.[57].

\section{Acknowledgements}

Authors thank the French National Research Agency for its support through the Labex STORE-EX project (ANR-10LABX-76-01). This work was performed using HPC resources from GENCI-CINES (Grant 2019-A0060910369). 
1. Cheng, X.-B., Zhang, R., Zhao, C.-Z. \& Zhang, Q. Toward Safe Lithium Metal Anode in Rechargeable Batteries: A Review. Chem. Rev. 117, 10403-10473 (2017).

2. Zhang, J.-G., Xu, W. \& Henderson, W. A. Lithium Metal Anodes and Rechargeable Lithium Metal Batteries. vol. 249 (Springer International Publishing, 2017).

3. Xu, X. et al. Recent progresses in the suppression method based on the growth mechanism of lithium dendrite. J. Energy Chem. 27, 513-527 (2018).

4. Wang, L. et al. Engineering of lithium-metal anodes towards a safe and stable battery. Energy Storage Mater. 14, 22-48 (2018).

5. Wang, D. et al. Towards High-Safe Lithium Metal Anodes: Suppressing Lithium Dendrites via Tuning Surface Energy. Adv. Sci. 4, 1600168 (2017).

6. Tikekar, M. D., Choudhury, S., Tu, Z. \& Archer, L. A. Design principles for electrolytes and interfaces for stable lithium-metal batteries. Nat. Energy 1, 16114 (2016).

7. Obrovac, M. N. \& Chevrier, V. L. Alloy Negative Electrodes for Li-lon Batteries. Chem. Rev. 114, 1144411502 (2014).

8. Rehnlund, D. et al. Lithium trapping in alloy forming electrodes and current collectors for lithium based batteries. Energy Environ. Sci. 10, 1350-1357 (2017).

9. Cheng, X.-B. et al. A Review of Solid Electrolyte Interphases on Lithium Metal Anode. Adv. Sci. 3, 1500213 (2016).

10. Fan, X. et al. Highly Fluorinated Interphases Enable High-Voltage Li-Metal Batteries. Chem 4, 174-185 (2018).

11. Tu, Z., Nath, P., Lu, Y., Tikekar, M. D. \& Archer, L. A. Nanostructured Electrolytes for Stable Lithium Electrodeposition in Secondary Batteries. Acc. Chem. Res. 48, 2947-2956 (2015).

12. Yuan, Y. et al. Regulating Li deposition by constructing LiF-rich host for dendrite-free lithium metal anode. Energy Storage Mater. 16, 411-418 (2019). 
13. Ding, F. et al. Dendrite-Free Lithium Deposition via Self-Healing Electrostatic Shield Mechanism. J. Am. Chem. Soc. 135, 4450-4456 (2013).

14. Janek, J. \& Zeier, W. G. A solid future for battery development. Nat. Energy 1, 16141 (2016).

15. Gao, J., Zhao, Y.-S., Shi, S.-Q. \& Li, H. Lithium-ion transport in inorganic solid state electrolyte. Chin. Phys. B 25, 018211 (2016).

16. Chen, R., Qu, W., Guo, X., Li, L. \& Wu, F. The pursuit of solid-state electrolytes for lithium batteries: from comprehensive insight to emerging horizons. Mater. Horiz. 3, 487-516 (2016).

17. Thangadurai, V., Narayanan, S. \& Pinzaru, D. Garnet-type solid-state fast Li ion conductors for Li batteries: critical review. Chem. Soc. Rev. 43, 4714 (2014).

18. Tarascon, J.-M., Gozdz, A. S., Schmutz, C., Shokoohi, F. \& Warren, P. C. Performance of Bellcore's plastic rechargeable Li-ion batteries. Solid State Ion. 86-88, 49-54 (1996).

19. Nishida, T., Nishikawa, K., Rosso, M. \& Fukunaka, Y. Optical observation of Li dendrite growth in ionic liquid. Electrochimica Acta 100, 333-341 (2013).

20. Lu, D. et al. Failure Mechanism for Fast-Charged Lithium Metal Batteries with Liquid Electrolytes. Adv. Energy Mater. 5, 1400993 (2015).

21. Crowther, O. \& West, A. C. Effect of Electrolyte Composition on Lithium Dendrite Growth. J. Electrochem. Soc. 155, A806 (2008).

22. Bai, P., Li, J., Brushett, F. R. \& Bazant, M. Z. Transition of lithium growth mechanisms in liquid electrolytes. Energy Environ. Sci. 9, 3221-3229 (2016).

23. Yu, S.-H., Huang, X., Brock, J. D. \& Abruña, H. D. Regulating Key Variables and Visualizing Lithium Dendrite Growth: An Operando X-ray Study. J. Am. Chem. Soc. 141, 8441-8449 (2019).

24. Bai, P. et al. Interactions between Lithium Growths and Nanoporous Ceramic Separators. Joule 2, 2434-2449 (2018).

25. Steiger, J. Mechanisms of Dendrite Growth in Lithium Metal Batteries. (2015). 
26. Chang, H. J. et al. Correlating Microstructural Lithium Metal Growth with Electrolyte Salt Depletion in Lithium Batteries Using ${ }^{7}$ Li MRI. J. Am. Chem. Soc. 137, 15209-15216 (2015).

27. Matsui, M. Study on electrochemically deposited Mg metal. J. Power Sources 196, 7048-7055 (2011).

28. Chen, K.-H. et al. Dead lithium: mass transport effects on voltage, capacity, and failure of lithium metal anodes. J. Mater. Chem. A 5, 11671-11681 (2017).

29. Hong, Y.-S. et al. In operando observation of chemical and mechanical stability of Li and $\mathrm{Na}$ dendrites under quasi-zero electrochemical field. Energy Storage Mater. 11, 118-126 (2018).

30. Rodriguez, R. et al. In Situ Optical Imaging of Sodium Electrodeposition: Effects of Fluoroethylene Carbonate. ACS Energy Lett. 2, 2051-2057 (2017).

31. Medenbach, L. et al. Origins of Dendrite Formation in Sodium-Oxygen Batteries and Possible Countermeasures. Energy Technol. 5, 2265-2274 (2017).

32. Wei, S. et al. Highly Stable Sodium Batteries Enabled by Functional Ionic Polymer Membranes. Adv. Mater. 29, 1605512 (2017).

33. Sand, H. J. S. III. On the concentration at the electrodes in a solution, with special reference to the liberation of hydrogen by electrolysis of a mixture of copper sulphate and sulphuric acid. Lond. Edinb. Dublin Philos. Mag. J. Sci. 1, 45-79 (1901).

34. Bard, A. J. \& Faulkner, L. R. Electrochemical methods: fundamentals and applications. (Wiley, 2001).

35. Wranglen, G. Dendrites and growth layers in the electrocrystallization of metals. 2, 130-146 (1960).

36. Davidson, R. et al. Formation of Magnesium Dendrites during Electrodeposition. ACS Energy Lett. 4, 375-376 (2019). 
37. Davidson, R. et al. Mapping mechanisms and growth regimes of magnesium electrodeposition at high current densities. Mater. Horiz. 10.1039.C9MH01367A (2020) doi:10.1039/C9MH01367A.

38. Mayers, M. Z., Kaminski, J. W. \& Miller, T. F. Suppression of Dendrite Formation via Pulse Charging in Rechargeable Lithium Metal Batteries. J. Phys. Chem. C 116, 26214-26221 (2012).

39. Aryanfar, A. et al. Dynamics of Lithium Dendrite Growth and Inhibition: Pulse Charging Experiments and Monte Carlo Calculations. J. Phys. Chem. Lett. 5, 1721-1726 (2014).

40. Aryanfar, A. et al. Thermal relaxation of lithium dendrites. Phys. Chem. Chem. Phys. 17, 80008005 (2015).

41. Ozhabes, Y., Gunceler, D. \& Arias, T. A. Stability and surface diffusion at lithium-electrolyte interphases with connections to dendrite suppression. ArXiv150405799 Cond-Mat Physicsphysics (2015).

42. Jäckle, M. \& Groß, A. Microscopic properties of lithium, sodium, and magnesium battery anode materials related to possible dendrite growth. J. Chem. Phys. 141, 174710 (2014).

43. Hörmann, N. G. et al. Some challenges in the first-principles modeling of structures and processes in electrochemical energy storage and transfer. J. Power Sources 275, 531-538 (2015).

44. Aryanfar, A. et al. Annealing kinetics of electrodeposited lithium dendrites. J Chem Phys 6 (2015). 45. Jackle, M., Helmbrecht, K., Smits, M., Stottmeister, D. \& Groß, A. Self-diffusion barriers: possible descriptors for dendrite growth in batteries? Environ. Sci. 9 (2018).

46. Gaissmaier, D., Fantauzzi, D. \& Jacob, T. First principles studies of self-diffusion processes on metallic lithium surfaces. J. Chem. Phys. 150, 041723 (2019).

47. Hagopian, A., Kopač, D., Filhol, J.-S. \& Kopač Lautar, A. Morphology evolution and dendrite growth in Li- and Mg-metal batteries: A potential dependent thermodynamic and kinetic multiscale ab initio study. Electrochimica Acta 353, 136493 (2020). 
48. Qin, X., Shao, M. \& Balbuena, P. B. Elucidating mechanisms of Li plating on Li anodes of lithiumbased batteries. Electrochimica Acta 284, 485-494 (2018).

49. Kamphaus, E. P. et al. Role of Inorganic Surface Layer on Solid Electrolyte Interphase Evolution at Li-Metal Anodes. ACS Appl. Mater. Interfaces 11, 31467-31476 (2019).

50. Ely, D. R. Phase field kinetics of lithium electrodeposits. J. Power Sources 14 (2014).

51. Chen, L. et al. Modulation of dendritic patterns during electrodeposition: A nonlinear phase-field model. J. Power Sources 300, 376-385 (2015).

52. Zhang, R., Shen, X., Cheng, X.-B. \& Zhang, Q. The dendrite growth in 3D structured lithium metal anodes: Electron or ion transfer limitation? Energy Storage Mater. S2405829719302247 (2019) doi:10.1016/j.ensm.2019.03.029.

53. Kopač Lautar, A., Hagopian, A. \& Filhol, J.-S. Modeling interfacial electrochemistry: concepts and tools. Phys. Chem. Chem. Phys. 22, 10569-10580 (2020).

54. Filhol, J.-S. \& Neurock, M. Elucidation of the Electrochemical Activation of Water over Pd by First Principles. Angew. Chem. Int. Ed. 45, 402-406 (2006).

55. Mamatkulov, M. \& Filhol, J.-S. An abinitio study of electrochemical vs. electromechanical properties: the case of CO adsorbed on a Pt(111) surface. Phys. Chem. Chem. Phys. 13, 7675 (2011).

56. Filhol, J.-S. \& Doublet, M.-L. An ab initio study of surface electrochemical disproportionation: The case of a water monolayer adsorbed on a Pd(111) surface. Catal. Today 202, 87-97 (2013).

57. Lespes, N. \& Filhol, J.-S. Using Implicit Solvent in Ab Initio Electrochemical Modeling: Investigating $\mathrm{Li}^{+} /$Li Electrochemistry at a Li/Solvent Interface. J. Chem. Theory Comput. 11, 33753382 (2015).

58. Lespes, N. \& Filhol, J.-S. Using the electrochemical dimension to build water/Ru(0001) phase diagram. Surf. Sci. 631, 8-16 (2015). 
59. Kopač Lautar, A., Kopač, D., Rejec, T., Bančič, T. \& Dominko, R. Morphology evolution of magnesium facets: DFT and KMC simulations. Phys. Chem. Chem. Phys. 21, 2434-2442 (2019).

60. Lippmann, M. G. Relations entre les phénomènes électriques et capillaires. Ann. Chim. Phys. 494-549 (1875).

61. Filhol, J.-S. \& Doublet, M.-L. Conceptual Surface Electrochemistry and New Redox Descriptors. J. Phys. Chem. C 118, 19023-19031 (2014).

62. Schmickler, W. Interfacial electrochemistry. (Oxford University Press, 1996).

63. Kamrani Moghaddam, L., Ramezani Paschepari, S., Zaimy, M. A., Abdalaian, A. \& Jebali, A. The inhibition of epidermal growth factor receptor signaling by hexagonal selenium nanoparticles modified by SiRNA. Cancer Gene Ther. 23, 321-325 (2016).

64. Faglioni, F., Merinov, B. V. \& Goddard, W. A. Room-Temperature Lithium Phases from Density Functional Theory. J. Phys. Chem. C 120, 27104-27108 (2016).

65. Kamrani Moghaddam, L., Ramezani Paschepari, S., Zaimy, M. A., Abdalaian, A. \& Jebali, A. The inhibition of epidermal growth factor receptor signaling by hexagonal selenium nanoparticles modified by SiRNA. Cancer Gene Ther. 23, 321-325 (2016).

66. Kokko, K., Salo, P. T., Laihia, R. \& Mansikka, K. Work function and surface energy of optimized lithium slabs. Phys. Rev. B 52, 1536-1539 (1995).

67. Kim, I.-T., Egashira, M., Yoshimoto, N. \& Morita, M. Effects of electrolytic composition on the electric double-layer capacitance at smooth-surface carbon electrodes in organic media. Electrochimica Acta 55, 6632-6638 (2010).

68. Mathew, K., Kolluru, V. S. C., Mula, S., Steinmann, S. N. \& Hennig, R. G. Implicit self-consistent electrolyte model in plane-wave density-functional theory. J. Chem. Phys. 151, 234101 (2019).

69. Garza, A. J., Bell, A. T. \& Head-Gordon, M. Mechanism of CO2 Reduction at Copper Surfaces: Pathways to C2 Products. ACS Catal 10 (2018). 
70. Gauthier, J. A. et al. Challenges in Modeling Electrochemical Reaction Energetics with Polarizable Continuum Models. ACS Catal 12 (2019).

71. Lithium-ion batteries: solid-electrolyte interphase. (Imperial College Press, 2007).

72. Li, Y. et al. Atomic structure of sensitive battery materials and interfaces revealed by cryoelectron microscopy. Science 358, 506-510 (2017).

73. Theory of the Inhomogeneous Electron Gas. (Springer US, 1983). doi:10.1007/978-1-4899-04157.

74. He, Y. et al. Origin of lithium whisker formation and growth under stress. Nat. Nanotechnol. 14, 1042-1047 (2019).

75. Kopač Lautar, A. et al. Electrolyte Reactivity in the Double Layer in Mg Batteries: An Interface Potential-Dependent DFT Study. J. Am. Chem. Soc. 142, 5146-5153 (2020).

76. Shi, L., Xu, A. \& Zhao, T. First-Principles Investigations of the Working Mechanism of 2D $h$-BN as an Interfacial Layer for the Anode of Lithium Metal Batteries. ACS Appl. Mater. Interfaces 9, 19871994 (2017).

77. Yan, K. et al. Ultrathin Two-Dimensional Atomic Crystals as Stable Interfacial Layer for Improvement of Lithium Metal Anode. Nano Lett. 14, 6016-6022 (2014).

78. Fabbris, G. \& Hafner, J. Ab initio molecular dynamics for liquid metals. Phys Rev B 47, 558 (1993).

79. Kresse, G. \& Hafner, J. Ab initio molecular-dynamics simulation of the liquid-metal-amorphoussemiconductor transition in germanium. Phys Rev B 49, 14251 (1994).

80. Kresse, G. \& Furthmüller, J. Efficiency of ab-initio total energy calculations for metals and semiconductors using a plane-wave basis set. Comput Mat Sci 6, 15 (1996).

81. Kresse, G. \& Furthmüller, J. Efficient iterative schemes for ab initio total-energy calculations using a plane-wave basis set. Phys Rev B 54, 11169 (1996). 
82. Kresse, G. \& Joubert, D. From ultrasoft pseudopotentials to the projector augmented-wave method. Phys Rev B 59, 1758 (1999).

83. Blochl, P. E. Projector augmented-wave method. Phys Rev B 50, 17953 (1994).

84. Perdew, J. P., Burke, K. \& Ernzerhof, M. Generalized gradient approximation made simple. Phys. Rev. Lett. 77, 3865 (1996).

85. Mathew, K., Sundararaman, R., Letchworth-Weaver, K., Arias, T. A. \& Hennig, R. G. Implicit solvation model for density-functional study of nanocrystal surfaces and reaction pathways. J. Chem. Phys. 140, 084106 (2014). 